\title{
Development and optimization of a portable guidance system for the blind and visually impaired with the application of artificial intelligence
}

Tamas Nemes [guidewalk.geraet@gmail.com]

\begin{abstract}
This work describes a new type of portable, self-regulating guidance system, which learns to recognize obstacles with the help of a camera, artificial intelligence, and various sensors and thus warn the wearer through audio signals. For obstacle detection, a MobileNetV2 model with an SSD attachment is used which was trained on a custom dataset. Moreover, the system uses the data of motion and distance sensors to improve accuracy. Experimental results confirm that the system can operate with $74.9 \% \mathrm{mAP}$ accuracy and a reaction time of 0.15 seconds, meeting the performance standard for modern object detection applications. It will also be presented how those affected commented on the device and how the system could be transformed into a marketable product.
\end{abstract}

Keywords - Hearable Environment; Image Processing; Artificial Intelligence; Assistive Technologies; Blind Navigation; Visual Impairment; Guide System; Object Detection; Obstacle Avoidance; Convolutional Neural Network

\section{Contents}

1 Introduction $\quad 2$

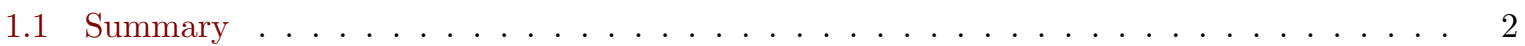

1.2 Related work . . . . . . . . . . . . . . . . . . . . . 2

2 Concept and structure $\quad 3$

2.1 Basic concept . . . . . . . . . . . . . . . . . . . . . . 3

2.2 Theoretical foundations for object detection networks . . . . . . . . . . . . . . 3

3 Method 4

3.1 Training and implementation of the object detection network . . . . . . . . . . . 4

3.2 Gesture recognition with the motion sensor . . . . . . . . . . . . . . . . 5

3.3 Distance measurement . . . . . . . . . . . . . . . . . . . . 6

3.4 Audio output programming . . . . . . . . . . . . . . . . . . . . . 7

3.5 Power management and casing design . . . . . . . . . . . . . . . . . . . . 7

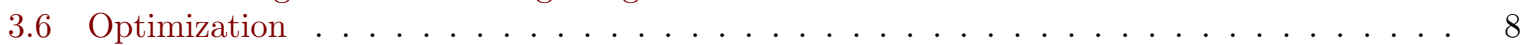

4 Results $\quad \mathbf{9}$

4.1 Accuracy and performance evaluation of the AI . . . . . . . . . . . . . . . . . . . . . . . . . .

4.2 Accuracy determination of the distance sensor . . . . . . . . . . . . . . . . . . . . . . . . . . . . . . . . .

4.3 Main loop execution speed and runtime evaluation . . . . . . . . . . . . . . . 10

5 Discussion $r \begin{aligned} & 11\end{aligned}$

6 Experiences of those affected $r$

7 Concepts for mass production $r$

8 Conclusion and prospects $r$ 


\section{Introduction}

\subsection{Summary}

The goal of this work is to develop a fully functional and user-friendly prototype for an AI-based guidance system for the blind called GUIDE-Walk, which should support the inclusion of visually impaired people. The device is based on a previous prototype model built in $2019^{1}$, which was equipped with basic functions such as distance measuring and real-time inference with a pre-trained object detection model. Although many measures have been taken to support the inclusion of the visually impaired in everyday traffic, those affected are still exposed to unexpected events and unknown obstacles in yet undiscovered areas. Small digital guidance systems can support the elaboration of self-reliance of its users, making them more aware of their surroundings and independent from costly methods like guide dogs. The proposed guidance system uses a camera to identify potential dangers and relevant objects in road traffic with object detection networks and notifies the blind wearer via audio warnings, as well as collects and uses additional information from a distance and a motion sensor to optimize performance. By receiving this regulated information about objects in the immediate vicinity and in the distance alike as well as about how far they are away, blind users can get a better picture of their surroundings including objects they otherwise won't have noticed. The device is hung around the neck with a loop and can be controlled via tilting gestures. A special focus lies on the following aspects:

- Implementation of the feedback from test subjects

- Focusing on a limited number of core functions and preferring quality over quantity

- Keeping the components' complexity at a minimum to ensure low costs in future industrial production

Code is available at: https://github.com/Totemi1324/GUIDE-Walk-v2.0

\subsection{Related work}

With AI being an indispensable part of today's society, many approaches to automated blind guidance systems have sprung up in recent years. In the early 2010s, the first working approaches were created, [7] and [3] just being a few examples. These rely on the analysis of camera images on the pixel level and the determination of key points with stereoscopic camera techniques. The algorithms can detect paths as well as moving objects in general. Because of the lack of computing power and fewer advancements in the field, mostly arithmetic methods were utilized instead of AI. In addition, hard-coded calculations like this are case-specific and very sensitive to little changes in the environment such as weather or different types of asphalt. After massive progress in artificial intelligence and its efficiency, papers such as [8] demonstrated approaches with utilizing image classification to recognize relevant scenes. The referenced paper uses a CIFAR-10 classification model on a Raspberry Pi to categorize an image into 10 classes, such as "car" or "building". Although they provide a far wider spectrum of accuracy and reliability, they are of limited use in guidance systems since classification networks are not capable of locating and identifying specific instances of obstacles, which means blind users have no perception of depth. Also, in an attempt to replace conventional guide dogs, robotic alternatives like [16] were invented, a dog-like robot on a leash that scans the environment using a 2D LiDAR. The work also has a great focus on machine-human interaction, an aspect very important in a guidance system one has to trust in. [14] and [17] are other robotic approaches that integrated this technology right into assistive devices already in use such as walkers or white canes. Both rather use pattern-recognition algorithms to detect objects and estimate poses. With Edge AI coming up as a future technology, companies like Microsoft $\mathbb{R}$ also designed free smartphone apps like Seeing $\mathrm{AI}^{\mathrm{TM}}$ [4], a very advanced program for recognizing faces and scenes, reading text and barcodes, and even perceiving color. The inference happens through the smartphone camera which requires the user to steadily hold the device. Only a small minority of these ideas were developed into marketable devices, examples being OrCam $\mathbb{R}$ MyEye $^{T M}$ [11] or InnoMake ${ }^{T M}$ [13], today's most developed assistance systems attachable to glasses or shoes. These devices cost between 2800-5800\$, thus limiting the number of affected people who can afford them drastically.

\footnotetext{
${ }^{1}$ The device started out as a project for the German Federal Young Researchers Competition where it achieved 1st place at regional level. The list of the winners can be retrieved from here: https://www. audi-mediacenter . com/de/publikationen/weitere/jugend-forscht-siegerliste-2020-888
} 


\section{Concept and structure}

\subsection{Basic concept}

The focus of the research was to develop a device that combines suitability for everyday use with high functionality and good performance and can therefore be turned into a product capable of competing with blind guidance devices that are on the market today. The prototype from 2019 was based entirely on Python code, which was easy to use to get started, but in comparison is very inefficient and hardly suitable for mass production.

Therefore, this concept should be realized using $\mathrm{C}++$ code only. The main advantages of $\mathrm{C}++$ over Python are mainly a shorter runtime and better control over memory management, which is why these aspects are mainly optimized in the code. Furthermore, $\mathrm{C}++$ provides many opportunities for the usage of external libraries, e.g. OpenCV for image processing and Boost for logging, since most of these widely used applications utilize $\mathrm{C}++$ in the backend already. A great aspect of software development is to find and implement suitable libraries for all functionalities and components.

Fig. 1 illustrates all the components of the device prototype with a corresponding designation.

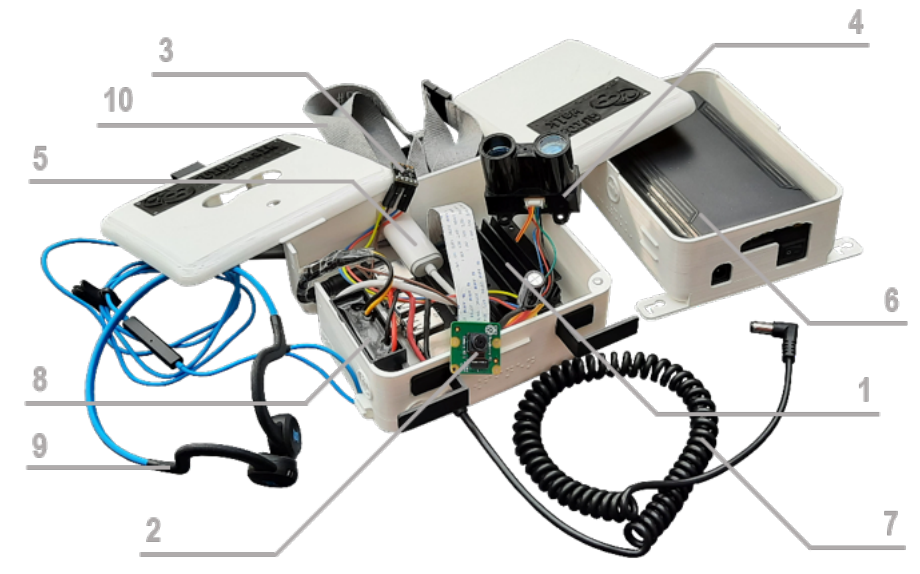

1: Jetson Nano ${ }^{\mathrm{TM}}$

2: Raspberry Pi Camera v2.1

3: MPU9250 and BME280

breakout board

4: LiDAR-Lite 33

5: External sound card

6: 12V/6A powerbank

7: $2,1 \mathrm{~mm} / 5,5 \mathrm{~mm}$ DC-cable

8: $5 \mathrm{~V}$ buck converter

9: Bone conduction

headphones

10: Neck loop

Figure 1: Components of the prototype, numbered

\subsection{Theoretical foundations for object detection networks}

As indicated, AI plays a key role in this application and all other components were designed to be centered around and to complement it in a meaningful way. To better understand the functionality of the model used in the program, the structure with the key components will be briefly explained below.

In the 2019 prototype, a pre-trained model of the MobileNetV2 network was used, which was controlled by the Nvidia@ Jetson Inference library. An object detection network suits the application best, as it can detect the position of relevant objects in the image with bounding boxes. The requirements were that the network should be as fast and, at the same time, as accurate as possible. Also, it should be controllable via $\mathrm{C}++$. After researching the AI model mentioned before, the SSD architecture by Wei Liu et al. proved to be most suitable, which is explained in detail in [10] and combines the properties referred to above.

SSD stands for "Single Shot MultiBox Detector" (Fig. 2) and describes a type of Convolutional Neural Network (CNN) that does not use fully-connected layers and, therefore, only needs one pass to make detections. It consists of two main parts: the support network and the SSD add-on. The latter is common to all SSD models, while the former can theoretically be replaced by any network.

The support network, which is VGG-16 in the schematic, is a slightly adapted CNN and largely determines the speed and accuracy of the entire network. For this application, MobileNetV2, the second iteration of the network originally described in [6], has a good balance between accuracy, model size, and number of operations, which are important for the use on edge devices. These numbers can be observed in Tab. 1, which was adapted from [6] and shows the competitive ImageNet accuracy with relatively few parameters. 
MobileNetV2 is a relatively new and fast CNN made up of convolution and pooling layers and specifically created for mobile vision applications. Its task is to generate a feature map from the input, a matrix that summarizes the results of all pattern comparisons of the convolution layers. Then the SSD addi-

\begin{tabular}{l|l|l|l} 
Model & $\begin{array}{l}\text { ImageNet } \\
\text { accuracy }\end{array}$ & $\begin{array}{l}\text { Million } \\
\text { Mult-Adds }\end{array}$ & $\begin{array}{l}\text { Million } \\
\text { parameters }\end{array}$ \\
\hline \hline MobileNet-224 & $70.6 \%$ & 569 & 4.2 \\
GoogleNet & $69.8 \%$ & 1550 & 6.8 \\
VGG-16 & $71.5 \%$ & 15300 & 138
\end{tabular}

Table 1: MobileNet in comparison to popular models, adapted from [6] tion divides the output of the support network to a grid, the resolution of which becoming smaller and smaller as the inferencing proceeds. The innovative aspect of SSD is that a certain number of bounding boxes with pre-determined aspect ratios are created in each grid cell. Layer Conv4_3, for example, divides the output to a grid of size $38 \times 38$, and makes 4 predictions in each cell (hence "MultiBox"), thereby creating a fixed number of boxes $\left(N_{o}=\right.$ number of output boxes, $b_{c}=$ boxes per cell, $s=$ grid size):

$$
N_{o}=b_{c} \times s^{2}=4 \times 38^{2}=5776
$$

The final network output yields a total of 8732 detections per class. Therefore, a non-maxima suppression is necessary to filter duplicates or boxes that probably belong to the same object. Finally, the detections are sorted according to confidence, outputting the top 100.

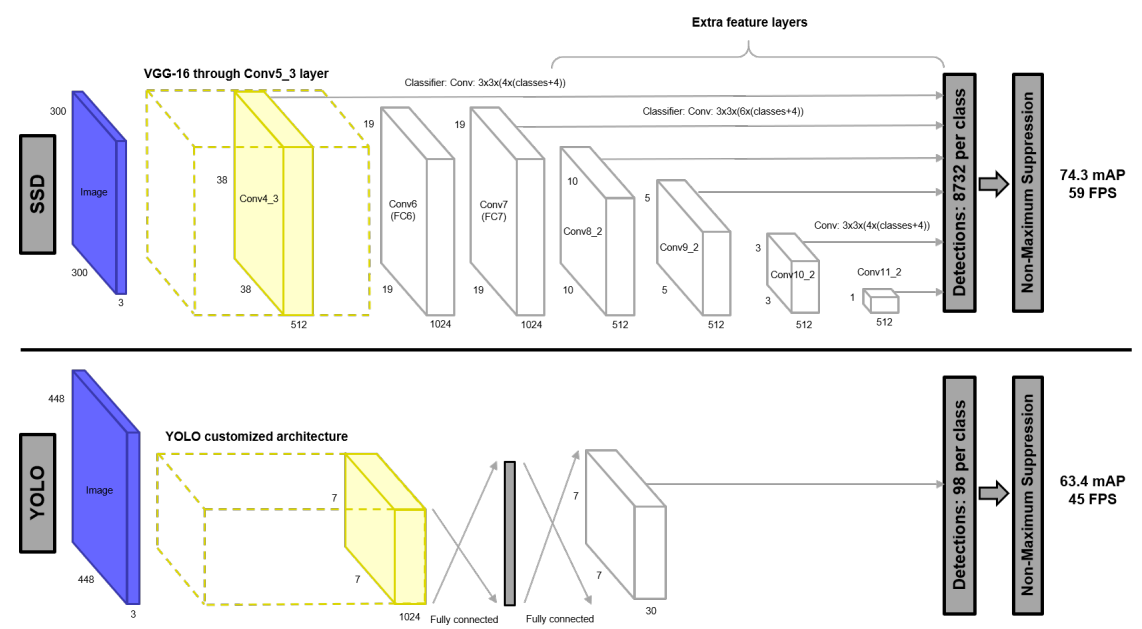

Figure 2: Schematic of SSD in comparison to the state-of-the-art network YOLO, adapted from [10]

\section{Method}

\subsection{Training and implementation of the object detection network}

One of the main reasons for the decision in favor of MobileNet-SSD v2 was its $\mathrm{C}++$ compatibility. An implementation of the network for the deep learning framework Caffe can be found on GitHub [18], which is also C-based and whose networks can be used for inference with the lightweight OpenCV DNN module (net.h).

The network should be able to recognize ten different objects in the images captured by the camera: pedestrians, cars, buses, bicycles, motorcycles, benches, chairs, garbage cans, and red and green pedestrian lights. To ensure the best possible performance, it is necessary to train the network with these classes only. But since there is no ready-to-use dataset just for these classes, an own had to be created. Using custom Python scripts, the images of the required classes and the annotations from the MS-COCO [9] and Pascal-VOC [5] datasets were extracted, converting them into the XML format. 
Also, the Ampelpilot [12] dataset was added, created by teams of the Hochschule Augsburg and the University of Tübingen for recognizing German pedestrian traffic lights (which was relevant because of the different appearance of American models). Finally, own recordings were contributed by sorting and labeling them with the LabelImg tool. In total, 46,512 pictures came together, of which 24,686 are from COCO and VOC and 21,826 are own ones (total size: approx. 8GB). The distribution of classes among the images is shown in Fig. 3. Both the Python code and the dataset, as well as the AI configuration files, can be viewed and downloaded from the GitHub repo. For pre-training and finetuning, the Caffe framework was used on a high-performance computer (CPU: AMDR Ryzen 9 3950X with 32 threads; GPU: Nvidiaß) RTX 3070; RAM: 32GB). This computing capacity was able to achieve consistent results. By applying a training split of $0.85 \%$ for the train-val and $0.15 \%$ for the test dataset, the network was trained with 39,535 and tested with 6,977 images. The separation is done automatically by scripts to ensure that the test set does not contain images from the training set.

\subsection{Gesture recognition with the motion sensor}

In addition to the AI, which makes up the core of the device, several sensors are used to make object detection more secure and extend it with additional functionality. A GPIO extension header with 40 pins is integrated on the Jetson Nano board to which these can be connected. It has two I2C, two SPI, and one UART bus and is almost identical to that of the Raspberry Pi in terms of pin assignment.

To have more options for detecting movement on the road, a motion sensor is integrated into the device's housing. The sensor was required to be $\mathrm{C}++$ compatible and be easily accessible using the Jetson $\mathrm{Nano}^{\mathrm{TM}}$ ' $\mathrm{s}$ integrated libraries for communication via GPIO pins. The 3-axis sensor MPU9250 by InvenSense@ with integrated accelerometer, gyroscope, and magnetometer and the BME280 temperature and pressure

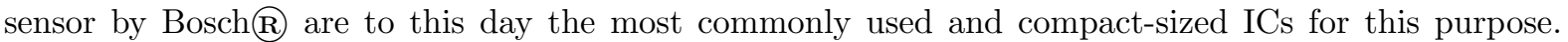
They are connected with jumper cables over one $\mathrm{I}^{2} \mathrm{C}$ bus only using a breakout board with an additional EM7180 motion co-processor and an M24512DFC EEPROM from Tindie (usfs_master.h). In Fig. 4 the connection of the components can be seen in detail.

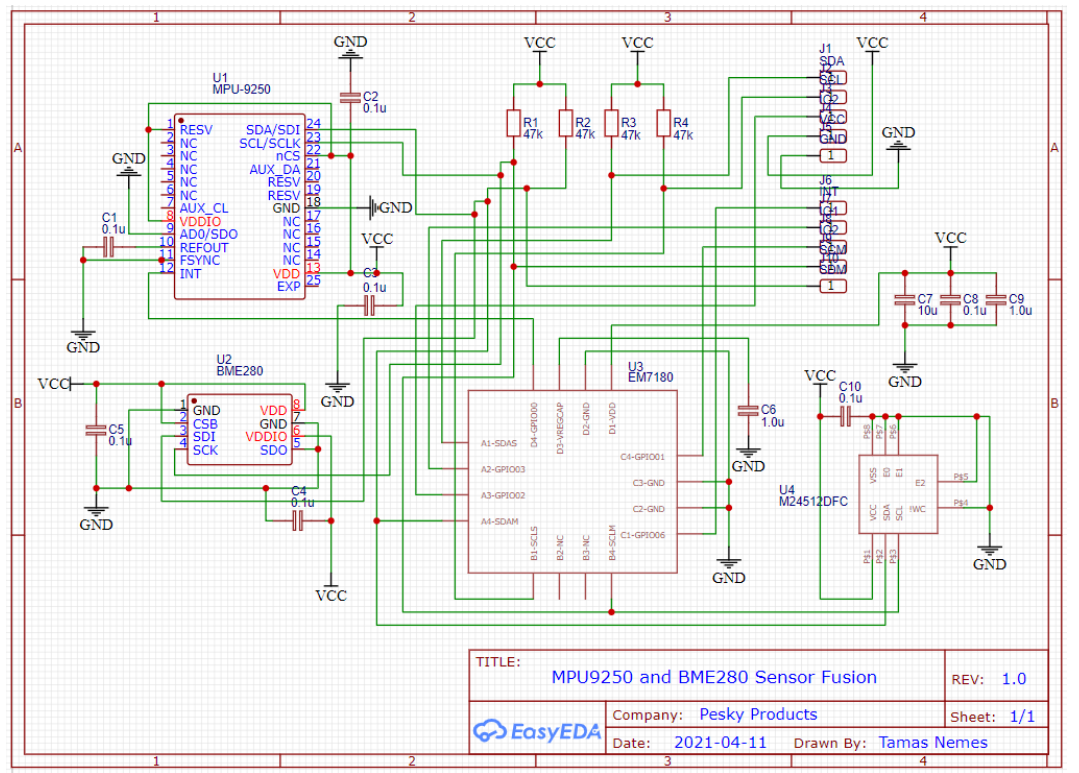

Figure 4: Schematic sheet of the MPU9250 and BME280 sensor fusion 
With the measurements retrieved from the breakout, additional data can be calculated. The primary data output from the EM7180 are quaternions $\left(q_{w}, q_{x}, q_{y}\right.$, and $\left.q_{z}\right)$, which uniquely define device orientation and can be converted to the Euler angles roll (2), pitch (3), and yaw (4) to determine the absolute orientation in space, as proved by [2]:

$$
\begin{gathered}
\phi=\arctan 2\left[2\left(q_{w} \times q_{x}+q_{y} \times q_{z}\right), q_{w}^{2}-q_{x}^{2}-q_{y}^{2}+q_{z}^{2}\right] \\
\theta=-\arcsin \left[2\left(q_{x} \times q_{z}-q_{w} \times q_{y}\right) \times \frac{180}{\pi}\right] \\
\psi=\arctan 2\left[2\left(q_{x} \times q_{y}+q_{w} \times q_{z}\right), q_{w}^{2}+q_{x}^{2}-q_{y}^{2}-q_{z}^{2}\right] \times \frac{180}{\pi}+13.8
\end{gathered}
$$

The BME280 also returns data on atmospheric pressure, from which the altitude above sea level can be estimated with the formula (5) derived from the International Standard Atmosphere defined in [1]. $p$ denotes the measured pressure in $\mathrm{hPa}$ and $p_{0}$ is equal to the pressure at sea level $(1013.25 \mathrm{hPa})$ :

$$
h=\left[1-\left(\frac{p}{p_{0}}\right)^{\frac{1}{5.255}}\right] \times 44330
$$

The sensors are applied in a wide variety of use cases in the device. The idea of programming tap detection already existed in the prototype phase to enable the user to interact intuitively with the device through tapping gestures, for example, to call up information about the surroundings. After gathering sample data and plotting them in graphs, it became evident that the MPU9250 accelerometer is not suitable for a reliable tap detection algorithm since the measured values were impaired by unintentional movements and a much too great noise during walking. Therefore, gesture control was solved with the Euler angles instead. The wearer can turn the device in different angles and use it to perform four gestures: tilt to the right for information on time, temperature and altitude, to the left to activate standby mode, and up to switch off the device, which must be executed twice to confirm it. Furthermore, the distance feedback can be deactivated with a downward tilt. A gesture is registered whenever a certain threshold value for the alignments is exceeded, which is 60 degrees in any direction from the idle state. One of the most important functions of motion detection is to distinguish between walking and standing. This is used for constraining object recognition: pedestrian traffic lights are only recognized when the wearer stops to minimize false recognitions and thus to ensure more safety. To achieve this, the last 15 measurement values of the accelerometer are recorded in an array from which the standard deviation is calculated. This term describes how much the individual values deviate from their average and therefore indicate walking. Since the values are just samples and all different, the formula (6) for the sample standard deviation distribution [15] is used:

$$
s=\sqrt{\frac{1}{n-1} \times \sum_{i=1}^{n}\left(x_{i}-\bar{x}\right)^{2}}
$$

When walking, the spread of the measured values is relatively large, but if it falls below a threshold value, standing is assumed. The mean value of the height above sea level at two different points in time can be used to determine and warn of a sloping surface. Lastly, the camera's images are cropped using the y-axis of the gyroscope, which measures three-dimensional changes in angle. When the wearer rotates, the parts of the image that it rotates away from are insignificant and can be removed to optimize the performance of the AI. The amount of image that gets cropped depends on the rotation speed. All evaluations proved to be accurate after some minor optimizations.

\subsection{Distance measurement}

The distance sensor, namely an ultrasonic sensor, was already an integral part of the original prototype. This served the purpose of compensating for the weaknesses of the AI, which is recognizing not trained obstacles in the immediate vicinity. Similar to parking aids, the program should continuously measure the distance and give the wearer feedback through repeated signal tones as to whether they are approaching an obstacle and how far away it is.

However, the ultrasonic sensor (HC-SR04 by Sparkfun@) is struggling with significant accuracy problems on the Jetson system, which made it impossible to provide reliable distance feedback. While the 
functionality of the manufacturer's Arduino library can be transformed to run on the Jetson Nano ${ }^{T M}$, its execution requires superuser privileges and is much less precise because of the lags and delays caused by the operating system. After evaluating different sensors, the LiDAR Lite v3 by Garmin $\mathbb{R}$ has been chosen, which is a Time-of-Flight sensor with $\mathrm{I}^{2} \mathrm{C}$ communication and one of the cheaper LiDAR sensors on the market. It measures distances with a laser by determining the time between the pulse of the transmitter and the impact of the light on the detector. The instructions manual denotes a measurement accuracy of $\pm 2.5 \mathrm{~cm}(\approx 0.9 \mathrm{in})$ and a range of $40 \mathrm{~m}(\approx 131.2 \mathrm{ft})$ for the breakout module, which has a $\mathrm{C}++$ interface directly from the manufacturer (lidar.h).

\subsection{Audio output programming}

The output of audio signals, i.e. passing on the information that has been collected and processed by the individual components to the blind wearer, is an essential cornerstone of the system. To convey warnings in a user-friendly and resource-saving way, a text-to-speech (TTS) program was instructed to read them aloud, which then was recorded and edited for the device's program to play back. A total of 41 voicelines, that can be viewed in the GitHub repo, were created this way. For the distance feedback, sine waves are generated which are played back at certain time intervals. In the $\mathrm{C}++$ integration, audio files must be played asynchronously, i.e. parallel to the normal program sequence. The Mixer module of the Simple DirectMedia Layer (SDL) library loads the voicelines into memory and can play them in two channels simultaneously (audio.h). Since the Jetson Nano ${ }^{T M}$ does not have a headphone jack socket, an external sound card with a USB connection is required.

It soon became apparent, however, that the warnings generated by the program had to be filtered in some way, otherwise they would be output continuously and randomly. To organize them, warnings are first placed in a dynamic array, called a vector. Each warning itself is an array and has the following format:

$$
\left\{\left[\text { warning }_{i} d\right],[\text { state }],[\text { cooldown }],[\text { priority }]\right\}
$$

Each audio message has an ID (defined in the header file) and a priority value from 0-1000. The warnings are sorted according to priority and since all have different values, it's guaranteed that, if several messages are received at the same time, always the most important one can be found. For example, the system always warns of pedestrians or traffic lights rather than notifying the presence of benches or bins, and information about time and weather is immediately interrupted when nearby objects are detected. When an element of the vector has been played, its state is marked accordingly. From then on, a cooldown counter starts, after which the entry is removed. This is important because warnings are only saved to the queue if there is no other with the same ID in the list, which prevents an accumulation of warnings of the same type. Overall, this system helps to ensure that audio messages are sorted and that the wearer is not unduly overloaded with information.

\subsection{Power management and casing design}

Deciding on the way the device was worn and designing the casing in a way that made it comfortable and suitable for everyday use as well as practical and safe poses another challenge. After evaluating different ways of wearing the device, a method proved to be best where the final product is hung around the neck with a loop, ultimately because that way it can be put on and taken off much more quickly and easily, and the wearer is not restricted in the choice of clothing.

In contrast to the prototype, the battery has been moved to a separate box that fits in a bag or can be strapped to your belt. It can be removed using a sliding mechanism and is connected to the device via a spiral cable. The main box (see Fig. 5), which contains the Jetson $\mathrm{Nano}^{\mathrm{TM}}$ and the other components, is relatively

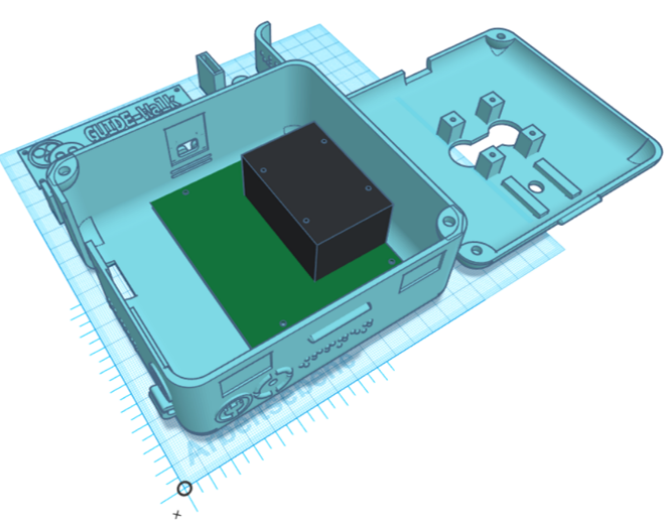

Figure 5: Final CAD casing design compact with dimensions of $12.9 \mathrm{~cm} \times 12 \mathrm{~cm} \times 6.4 \mathrm{~cm}$. It is equipped with an easily removable, but still firmly-fitting lid with a magnetic lock as well as recesses 
for the camera and the LiDAR sensor. On the lower half, there is a headphone socket, eyelets for the safety pins, and hooks for winding the cable. To make handling easier for blind people, all important points are marked with Braille or, if the wearer does not know Braille, with simple haptic symbols. This also applies to the container of the battery. The Jetson Nano is fixed in the housing with screws, which means that the device can be easily dismantled for repair purposes.

To supply the Jetson Nano with sufficient power in a mobile design, it was decided on providing it through the DC barrel jack. The power consumption is between 5-10W on paper, but in reality, it reaches higher levels such as the $5 \mathrm{~V}=2.1 \mathrm{~A}$ provided by USB are not sufficient. This has been confirmed

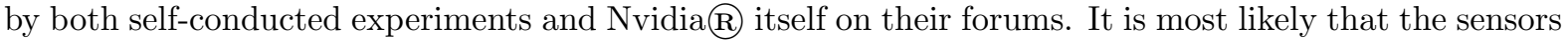
and peripherals are the cause of the additional power consumed. The DC socket $(5.5 \mathrm{~mm} / 2.1 \mathrm{~mm})$ can deliver up to $5 \mathrm{~V}=4 \mathrm{~A}$, i.e. double the current. To do this, the $\mathrm{J} 48$ pins must be short-circuited with a jumper. The power comes from a battery (capacity: $8300 \mathrm{mAh}$ ) that is easy to use for the blind and has a DC socket with an output of $12 \mathrm{~V}=6 \mathrm{~A}$. To step it down, a transformer was integrated into the housing.

\subsection{Optimization}

After the successful implementation of the individual modules, the task was to complete the program and optimize it as far as possible. The goal hereby is to achieve the shortest possible response time and, at the same time, the lowest possible energy consumption. With a few exceptions, these optimization processes run completely in the background and are self-contained.

This includes AI optimizations. In addition to reducing the model size with the previously described training, images that are unsuitable for evaluation are sorted out in advance. This is done by calculating the Laplace variance of the camera images, the value of which represents the degree of blurring. A build-in OpenCV function applies an edge-sensitive kernel to the image and the squared standard deviation of the results is calculated. If it falls below a certain threshold value, a new picture is taken. Since the image quality of the camera module suffers enormously when there is a lack of light, object recognition is deactivated at night to ensure more safety. The individual frames get reduced to a size of $64 p x \times 35 p x$, cropped to the upper third, and converted to the HSV color space. If the brightness, in this case, the mean of the "value" parameter of all pixels, is below 90 for 3 seconds, the AI pointer will be set to a nullpointer which switches off the AI. This will be communicated to the wearer as well. For increasing the speed of object detection, the AI calculations get carried out on the GPU, which significantly accelerates the process. The Jetson $\mathrm{Nano}^{\mathrm{TM}}$ 's integrated graphics chip with Maxwell architecture was specifically designed for this purpose, but to ensure stable operation, one additionally has to define a new energy profile for the board and limit the clock frequencies of the CPU (918MHz) and GPU $(640 \mathrm{MHz})$. Otherwise, the computer would crash due to excessive energy consumption and voltage undersupply ("brownout"). The performance was throttled somewhat as a result, but this measure was necessary to ensure consistent operation.

A very important tool for improving performance was multithreading. If the GStreamer pipeline delivers more images per second than the AI can process at the same time, the recordings accumulate and a time shift occurs between the just recorded frames and the ones already evaluated. To solve this fundamental problem, the camera stream is executed on a separate thread. Therefore, the thread produces a constant stream of 30 images per second, from which the main thread can copy the next image whenever necessary. Using this method (see Fig. 6), the recording rate of the camera is independent of the rest of the program.

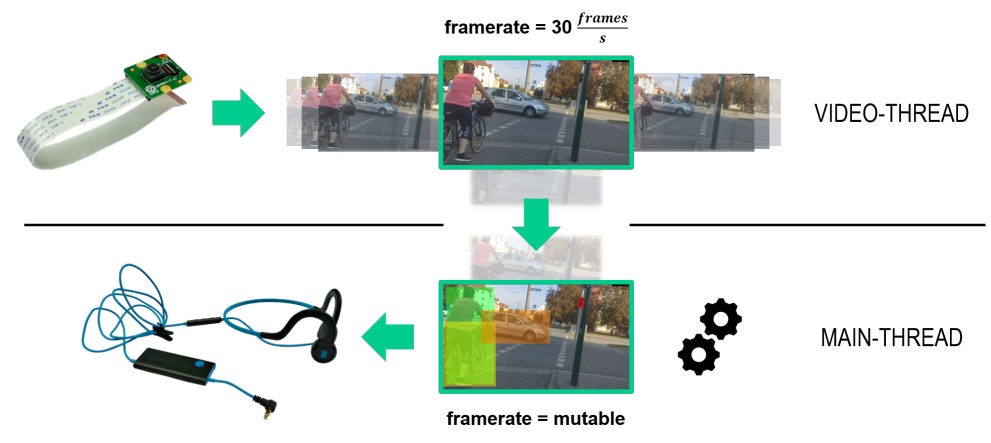

Figure 6: Schematic illustration of the program's structure using separate threads 
The distance measurement and feedback also run in a different thread, so that the time interval between the signal tones is not influenced by other factors. Crashes get prevented with implemented functions that detect errors in the sensors or other components, send a notification, and shut down the program in a controlled manner. All instances of all classes are emptied from the memory and the threads are terminated. Switching on and off is also designed to be as user-friendly as possible. By adding it to the autostart applications on Linux, the program runs automatically after the battery is plugged in. To shut down the device, two upward tilt gestures are required.

\section{Results}

\subsection{Accuracy and performance evaluation of the AI}

The final mAP (mean Average Precision) on the test dataset is $74.9 \%$ with an average loss of 2.43 calculated by Caffe. This is done by taking the mean AP score, calculated from the precision-recall curve, over all classes and a single IoU (Intersection over Union) threshold of 0.5. The final network only takes those predictions into account where it is more than $50 \%$ confident. In addition, a set of unrelated test images was created which show the individual objects in an isolated environment to obtain a more meaningful value. In Fig. 7, this result can be seen in comparison with other object detection networks that were trained using the same dataset and hardware.

Also, the performance of these networks in their $\mathrm{C}++$ implementation on the Jetson Nano ${ }^{\mathrm{TM}}$ was tested. A program script measured the duration of the individual inference runs per image, calculating an average value for CPU-time per frame. If the detections were rendered on the screen, this could be interpreted as the frame rate. The achieved FPS for the different models depending on the operating mode is shown in Fig. 8.

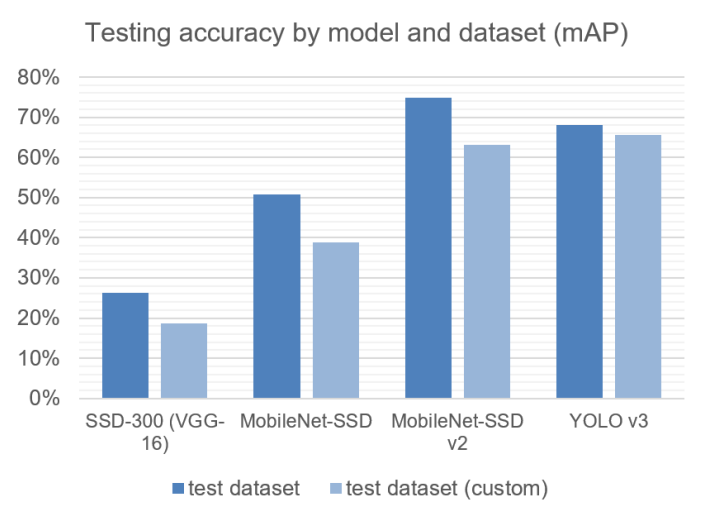

Figure 7: Testing accuracy by model and dataset (mAP)

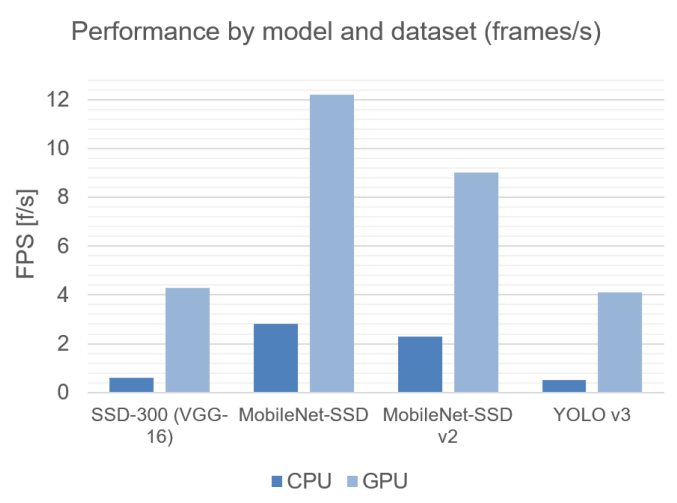

Figure 8: Performance by model and operating mode

\subsection{Accuracy determination of the distance sensor}

To test the accuracy, the sensor was attached at a distance of $30 \mathrm{~cm}$ in front of a wall, measuring the distance ten times per second for ten seconds. The same setup was repeated with a distance of $2 \mathrm{~m}$ and $5 \mathrm{~m}$. Fig. 9 shows the change in the measured values in the course of the measurements to the actual distance. 


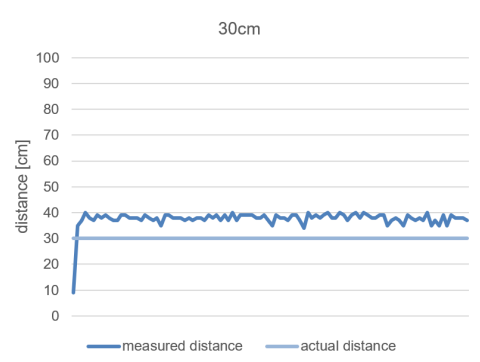

(a)

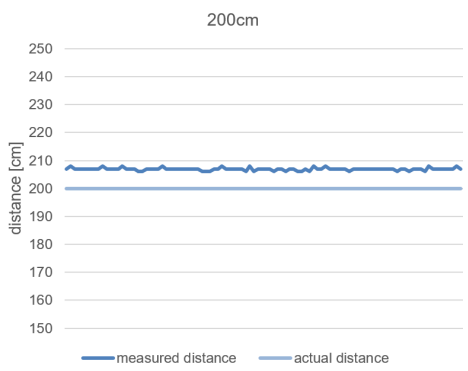

(b)

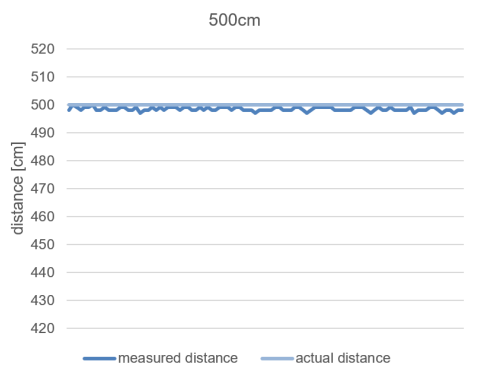

(c)

Figure 9: Results of the distance measurement experiments with a distance of (9a) 30cm, (9b) $2 \mathrm{~m}$ and $(9 \mathrm{c}) 5 \mathrm{~m}$.

\subsection{Main loop execution speed and runtime evaluation}

When the main program was finished, the system was tested as a whole so as to get a summary statistic of its performance and see how effective the optimizations mentioned above were. A simple FPS counter that uses the formula $F P S=\frac{t_{\text {end }}-t_{\text {start }}}{1,000,000 \mu s}$ measured the runtime of each loop execution over a period of 100 frames. The averaged data points are shown in Fig. 10, dependent on the hardware used for acceleration.

- With CPU only, the program achieves an average of 0.6 FPS.

- Using GPU acceleration, the frame rate rises to an average of 6.5 FPS.

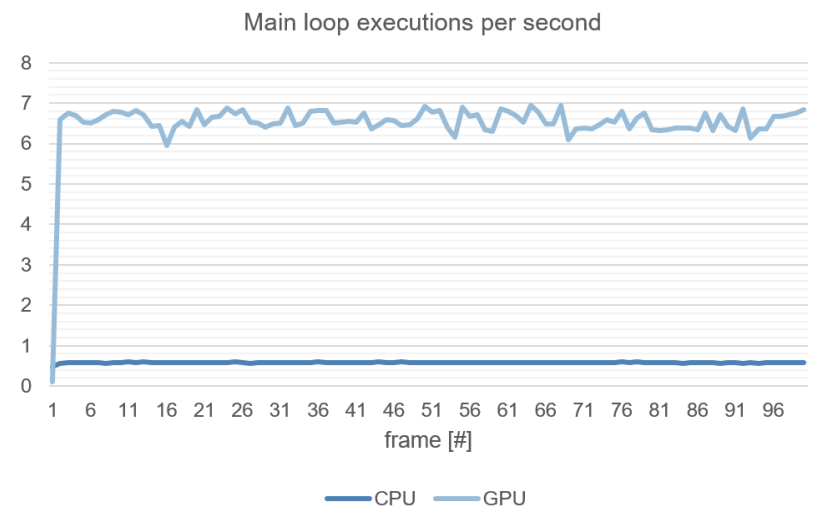

Figure 10: Results of the runtime test

When it comes to determining the battery life, there are two possible methods. Arithmetically, it can be calculated as follows:

$$
t_{\text {bat }}=\frac{U \times Q}{P}=\frac{5 \mathrm{~V} \times 8.3 \mathrm{Ah}}{10 \mathrm{~W}}=4.15 \mathrm{~h}
$$

An experimental determination was additionally performed to confirm the result. Using the LED lights on the power bank, you can observe at which pace it empties, measure the time and extrapolate it. Fig. 11 shows the results.

- With uninterrupted usage, the device runs for 7 to 8 hours.

- When not used, the device can be set to standby mode to increase battery life up to a factor of 1.25 .

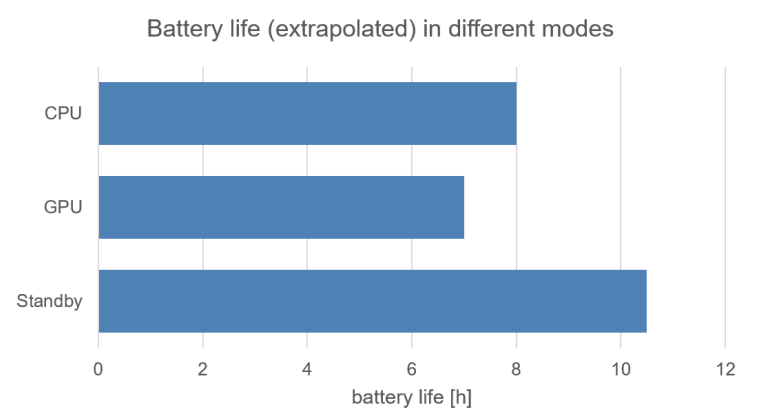

Figure 11: Battery life (extrapolated) in different modes 


\section{Discussion}

Of all tested networks, MobileNet-SSD v2 performs best. An accuracy percentage range of $70-80 \%$ is typical for modern object detection networks. This might seem low at first, but considering that the accuracy is calculated using the predictions' deviation from the exact positions of the original bounding boxes, it is not. To our human eyes, a network is considered "accurate" even if it roughly determines the location of objects in the image, which is why the achieved $\mathrm{mAP}$ of $74.9 \%$ is suitable for this application. By running a demo on some test images, a pattern of false boxes can be observed which are duplicates of larger boxes of the same object. Filtering them can lead to a further improvement of accuracy, which happens in post-processing and, therefore, cannot be measured by Caffe.

When it comes to execution time, MobileNet-SSD v2 did not achieve the best results, but since the accuracy of the fastest model is much lower, this limitation is justifiable. The frames per second were measured both with and without GPU acceleration, while the application of which quadrupled the results on average. Combining the operating speed of the AI inference and the overall program, the system achieves an average reaction time of $\frac{1}{6.5} s=0.15 s$. This is a great advantage compared to the reaction time of humans $(\approx 1 s)$. According to experiences of the test persons in the field testings, this time is not only completely sufficient but also more "forward-looking", especially if considered that the device is only meant to be used as a supplement for the perception of the blind.

The accuracy tests of the LiDAR sensor show that interestingly, the distance tends to be measured as too far, with this deviation from the actual value decreasing with increasing distance. At very short distances such as $30 \mathrm{~cm}$, the error can be up to $10 \mathrm{~cm}$, at $5 \mathrm{~m}$, on the other hand, the measurements are relatively accurate. In all three experiments, the measured values have a very small noise that does not affect performance. Therefore, one can assume that the sensor has a high level of accuracy since when walking, you are unlikely to reach a distance that is as small as $30 \mathrm{~cm}$.

The calculated battery life is imprecise and does not match the experimental results for several reasons: First, the power consumption of the Jetson Nano ${ }^{T \mathrm{M}}$ can only be estimated, and, second, physical parameters such as losses during transformation, etc. are not included in the formula. The experimentally determined duration, however, is a solid result that adequately covers most of the time when walking outside.

\section{Experiences of those affected}

While developing the project, it was particularly important to obtain first-hand feedback to improve aspects that most laypeople do not know of. For this purpose, after contacting various institutions for the blind, the device was tested by two visually impaired people in action on the street.

One of them sees object detection with artificial intelligence as a sensible approach when it comes to recognizing unexpected or sudden events such as obstacles or red lights. He also advocates the least possible impairment of the wearer's hearing. When it comes to the operation of the device, he criticizes that the system gave him too much and sometimes unreliable information. At his suggestion, the AI was improved with additional recordings and new training, and the curb detection was removed due to a shortage of more functional alternatives. Overall, the guidance device can contribute to the inclusion process in his opinion.

The other tester emphasizes that the assistance device can help with cuts by nearby pedestrians, such as hectic rush or (often unintentional) lack of consideration. He, therefore, sees the use of AI for object and traffic light detection as suitable but criticizes the inadequate filtering and the "flood" of information that is passed on to the wearer. In line with his proposal, the system for prioritizing warnings was revised. He also complained about the lack of a spatial conception of recognized hazards, which is why the distance measurement was linked to the object recognition to only issue a warning when an obstacle is in the immediate vicinity. In summary, the insights and findings from these discussions and tests were extremely helpful and have contributed significantly to improving the device in many ways. 


\section{Concepts for mass production}

It has been shown that the base concept of an AI-powered guidance system for traffic is functional and applicable. For the device to become a product that is available for everyone affected, however, the functionality of the used developer kits and breakout boards has to be transformed to a smaller and lighter package with more practicality. Based on the results presented, it is possible to deduce an informed guess on how this could be realized.

The highest level of functionality and speed is guaranteed if the device is designed as an embedded system on a custom printed circuit board (PCB) with selected components (Fig. 12, Fig. 13). A power-efficient microcontroller unit (MCU) with enough data ports to deal with the sensors and enough computing capacity for the various data streams in connection with a tensor processing unit (TPU) for AI acceleration would be a suitable pairing for this application. Microcontrollers like the STM32L476VGT6 or the RP2040 would fit this purpose most as both offer two $\mathrm{I}^{2} \mathrm{C}$ buses and allow for $\mathrm{C} / \mathrm{C}++$ development through software development kits (SDK). The recently released Google@ Coral $^{\mathrm{TM}} \mathrm{TPU}$ (G313-06329-00) could communicate with the MCU through USB and offers a complete developer toolkit as well. The sensor setup needs little changes, the layout of the EM7180 sensor fusion containing the accelerometer, magnetometer, gyroscope, and barometer, can be copied and integrated into the board as is. The remaining two sensors, the camera and the LiDAR, have to be on a separate breakout from the board since they need to face forward to capture the environment. To achieve minimum size and costs, inexpensive LiDAR chips can be utilized such as the VL53L5 by STR, which can also measure in a two-dimensional surface area by emitting an 8x8 laser grid instead of a one-directional beam. This data can be combined with the pictures taken by a camera module such as OV2640 to significantly improve the accuracy of the AI detections.

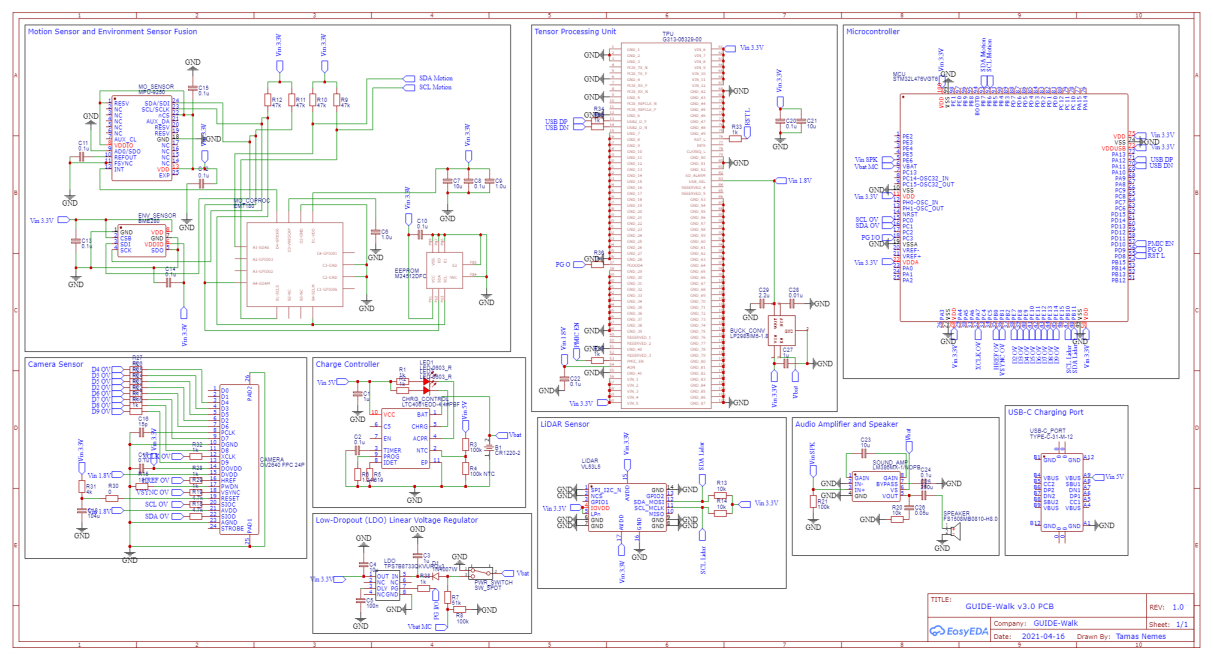

Figure 12: Schematic sketch of how the embedded system could look like

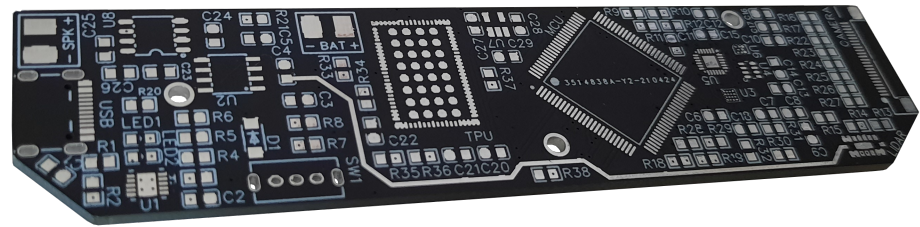

Figure 13: Sample PCB design based on Fig. 12, printed out 
All these parts need a voltage of $3.3 \mathrm{~V}$ to function and consume around $240 \mathrm{~mA}$ combined. Assuming a small-sized rechargeable Li-Ion battery with a capacity of 1000Ah is used to power the components, the battery life would be around 4 hours. Additionally, the system needs a voltage regulator and a charge controller for USB-C charging. Because of their compact sizes, the build parts could be integrated into wearable accessories such as glasses for additional comfort and discreetness. This enables the camera and LiDAR sensors to face to the front and the speakers to be hidden in the stems of the glasses as bone-conduction headphones.

Predicting the production cost and retail price in this stage of development is rather impossible because of the large number of factors that influence this process. However, by adding up the raw cost of the materials (Tab. 2), an estimated material price per unit of $77.14 \$$ can be cal-

\begin{tabular}{l|l|l} 
Component & Model number & $\begin{array}{l}\text { Expected } \\
\text { price/ } \text { unit }^{2}\end{array}$ \\
\hline \hline PCB & N.A. & $1.13 \$$ \\
\hline MCU & STM32L476VGT6 & $7.22 \$$ \\
\hline TPU & G313-06329-00 & $21.19 \$$ \\
\hline USB-C port & TYPE-C-31-M-12 & $0.19 \$$ \\
\hline Charge controller & LTC4061EDD\#PBF & $3.25 \$$ \\
\hline $\begin{array}{l}\text { LDO linear voltage } \\
\text { regulator }\end{array}$ & $\begin{array}{l}\text { TPS7B8733QKVUR } \\
\text { Q1R2 }\end{array}$ & $1.46 \$$ \\
\hline Sensor hub & EM7180V1CS16B+ & $1.50 \$$ \\
\hline $\begin{array}{l}\text { 9-axis motion sen- } \\
\text { sor }\end{array}$ & MPU9250 & $4.19 \$$ \\
\hline $\begin{array}{l}\text { Environment sen- } \\
\text { sor }\end{array}$ & BME280 & $2.80 \$$ \\
\hline $\begin{array}{l}\text { Time-of-Flight } \\
\text { ranging sensor }\end{array}$ & VL53L5CXV0GC/1 & $6.12 \$$ \\
\hline Camera module & OV2640 & $12.90 \$$ \\
\hline Audio amplifier & PAM8403DR & $0.39 \$$ \\
\hline Piezo transducers & CST-951RP-A & $1.30 \$$ \\
\hline Other components & $\begin{array}{l}\text { e.g. case parts, } \\
\text { screws, glue... }\end{array}$ & $13.50 \$$ \\
\hline \multicolumn{2}{|c}{ Raw material price/unit } & $77.14 \$$ \\
\hline Estimated production cost (materials only) & $77,140 \$$ \\
\hline
\end{tabular}
culated, assuming a quantity of 1000 units produced. It is crucial to keep in mind that these are rough estimates with no guarantee and additional, variable costs like direct labor, overhead costs, marketing and distribution costs, licenses, and certificates are not included. Since the target group is large enough to find enough buyers, the final product can likely be offered for less than $500 \$$, the price of a mid-range smartphone.

\section{Conclusion and prospects}

Now that the composition and functionality of the blind guide system have been explained in detail, it is safe to say that the goal of creating a navigation helper prototype for the blind has been achieved. In comparison to the previous model, the AI, in particular, has developed considerably and works much more precisely, and with the elaboration of new features and working speech output, it better represents a guide leading you through the traffic (although it is important to point out that the device does not replace a trained guide person). With further development, it is more than realistic that this project could make everyday life easier for the blind and visually impaired.

At the same time, the system is far from being perfect, and there are still numerous aspects of it waiting for improvements. As a creator of such software, one has a great responsibility towards its users. However, being open-minded and expectant about the future of guidance systems like the GUIDE-Walk is the best way to achieve further progress. In any case, we should continue stressing the fact that AI is used for helping people and perhaps give them a new perspective through the "eye of the computer".

\footnotetext{
${ }^{2}$ Pricing is taken from mouser.com, lcsc.com, omnipro.net and estore.st.com. The author does not guarantee for validity!
} 


\section{References}

[1] ISO 2533:1975. International Standard Atmosphere. Standard. Geneva, CH: International Organization for Standardization (ISO), 1975. URL: https://www.iso.org/standard/ 7472.html.

[2] Jose-Luis Blanco. A tutorial on SE(3) transformation parameterizations and on-manifold optimization. May 2013. URL: https ://citeseerx. ist.psu . edu/viewdoc/summary? doi=10.1.1.468.5407.

[3] David Castells, Joao Rodrigues, and J. du Buf. "Obstacle detection and avoidance on sidewalks". In: Proc. Int. Conf. on Computer Vision-Theory and Applications (VISAPP2010), Angers, France 2 (Jan. 2010), pp. 235-240. URL: https: //www . researchgate . net/ publication/216435190_Obstacle_detection_and_avoidance_on_sidewalks.

[4] Microsoft Corp. Seeing AI $I^{T M}$. URL: https://www.microsoft.com/en-us/ai/seeing-ai.

[5] Mark Everingham et al. "The Pascal Visual Object Classes (VOC) Challenge". In: International Journal of Computer Vision 88.2 (June 2010), pp. 303-338. ISSN: 1573-1405. DOI: 10.1007/s11263-009-0275-4. URL: https://doi.org/10.1007/s11263-009-0275-4.

[6] Andrew G. Howard et al. MobileNets: Efficient Convolutional Neural Networks for Mobile Vision Applications. 2017. arXiv: 1704.04861 [cs.CV].

[7] João José et al. "A vision system for detecting paths and moving obstacles for the blind". In: (Jan. 2010). URL: https : //www . researchgate . net/publication/216435222_A_ vision_system_for_detecting_paths_and_moving_obstacles_for_the_blind.

[8] Ashwani Kumar and Ankush Chourasia. "Blind Navigation System Using Artificial Intelligence". In: International Research Journal of Engineering and Technology (IRJET) 5.3 (Mar. 2018), pp. 601-605. URL: https : / / www . researchgate.net/publication/ 216435190_Obstacle_detection_and_avoidance_on_sidewalks.

[9] Tsung-Yi Lin et al. "Microsoft COCO: Common Objects in Context". In: (2014). Ed. by David Fleet et al., pp. 740-755. URL: https://doi .org/10.1007/978-3-319-10602$1 \_48$.

[10] Wei Liu et al. "SSD: Single Shot MultiBox Detector". In: CoRR abs/1512.02325 (2015), p. 4. arXiv: 1512.02325. URL: http://arxiv.org/abs/1512.02325.

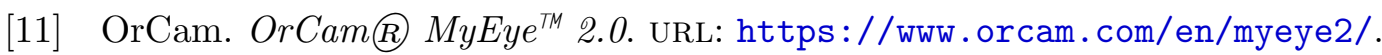

[12] patVlnta. Ampel-Pilot-Dataset on GitHub. URL: https://github.com/patVlnta/AmpelPilot-Dataset.

[13] Tec-Innovation. InnoMake ${ }^{T M}$. URL: https ://www . tec-innovation . com/en/innomakeen/.

[14] Andreas Wachaja et al. "Navigating blind people with walking impairments using a smart walker". In: Autonomous Robots 41.3 (Mar. 2017), pp. 555-573. ISSN: 1573-7527. DOI: 10.1007/s10514-016-9595-8. URL: https://doi.org/10.1007/s10514-016-9595-8.

[15] Eric W. Weisstein. "Standard Deviation". In: MathWorld-A Wolfram Web Resource (). URL: https://mathworld.wolfram.com/StandardDeviation.html.

[16] Anxing Xiao et al. Robotic Guide Dog: Leading a Human with Leash-Guided Hybrid Physical Interaction. 2021. arXiv: 2103.14300 [cs.RO]. URL: https://arxiv.org/abs/2103. 14300.

[17] Cang Ye et al. "Co-Robotic Cane: A New Robotic Navigation Aid for the Visually Impaired". In: IEEE Systems, Man, and Cybernetics Magazine 2.2 (2016), pp. 33-42. DOI: 10.1109/MSMC . 2015.2501167. 
[18] zhanghanbin3159. MobileNetV2-SSD on GitHub. URL: https://github.com/zhanghanbin3159/ MobileNetV2-SSD.

\section{Acknowledgments}

Many thanks to all those who supported and helped me in planning and realizing the project! These are in particular:

- My parents with the good ideas, their willingness to help, and their financial support for my project,

- The school of the Regensburger Domspatzen and its headmistress, Christine Lohse, without whose support my project would not have been possible,

- The Bavarian Young Researchers Sponsor Pool and EDIsys Kft., who provided me with a budget for the realization of the project,

- The testers Rainer Schliermann (Ostbayerische Technische Hochschule (OTH) Regensburg) and Rudolf Pichlmeier (Bavarian Association of the Blind and Visually Impaired (BBSB e.V.) Oberpfalz) with their cooperation, efforts and constructive criticism,

- The proof-readers and professional consultants René Grünbauer and Manfred Hofmann,

- The head of the Institution for the Blind in Regensburg, Ulrike Weimer, with her mediation work,

- Moritz Walker and Christoph Högl with their advice on technical issues,

- My friends Yannick Rittner, Patrick Soller, and Paul Kutzer with their spontaneous helpfulness. 